prednisone, antimalarials, and immunosuppressants, respectively.

Conclusions In a real-life cohort of SLE patients, clinical remission is consistently defined by applying either SLE-DAS, DORIS or Doria criteria. SLE-DAS definition is more straightforward, as it does not require the PGA or additional manifestations not included in SLEDAI.

\section{DO WE NEED PHYSICIAN GLOBAL ASSESSMENT FOR REMISSION IN SLE? ANALYSIS FROM AN SLE-COHORT AT A TERTIARY CENTER}

Johanna Mucke, Christina Düsing, Gamal Chehab, Matthias Schneider. Policlinic and Hiller Research Unit for Rheumatology, Heinrich-Heine-University Duesseldorf, Germany

\subsection{6/lupus-2020-eurolupus.28}

Background/Purpose The definition of an accurate target for a treat to target (T2T) approach in SLE has been challenging over the past years. Recently four definitions of remission were presented by the international DORIS task force comprising the parameters clinical activity (cSLEDAI), steroid dose, immunosuppressive therapy, serology and physician global assessment (PGA). In particular the PGA, its threshold and general utility have been and still are discussed controversially. It was our aim to evaluate the added value of PGA in remission assessment.

Methods In this monocentric cross-sectional study, patients with SLE according to the 1997 American College of Rheumatology (ACR) criteria were enrolled and assessed between September 2016 and December 2017. Two different definitions of remission were applied. The internationally accepted DORIS remission and a modified DORIS remission excluding PGA. Factors influencing PGA were assessed in the entire cohort. Regression analyses were used to establish differences between patients in DORIS and modified DORIS remission.

Results A total of 233 patients were included (87.6\% female). $98(41.9 \%)$ patients fulfilled any of the four DORIS remission definitions while $154(66.1 \%)$ patients were in modified remission (PGA was excluded). In general, PGA rating was dependent on disease activity (clinical SLEDAI; $\mathrm{p} \leq 0.0001$ ), depression (Center for Epidemiological Studies Depression Scale; $\mathrm{p}=0.049$ ), pain reported by the patient (numeric rating scale; $\mathrm{p} \leq 0.0001)$ and hypocomplementemia $(\mathrm{p} \leq 0.0001)$. Damage (SLICC damage index, SDI) did not influence PGA $(p=0.98)$. Both, DORIS and modified DORIS remission were associated with lower damage $(B=0.026$ vs $p=0.003)$, lower pain on NRS ( $p=0.001$ vs. $p=0.013)$, normal complement $(\mathrm{p}=0.0005$ vs $\mathrm{p}=0.005)$ and better illness perception $(p=0.006$ vs $p=0.023)$. Patients in modified DORIS remission tendentially received more often immunosuppressive therapy $(\mathrm{p}=0.046)$.

Conclusion Exclusion of PGA in remission assessment led to an increase of patients in remission. Clinical parameters and factors associated with DORIS remission vs. modified DORIS remission were similar, hence the added value of PGA in our cohort regarding remission assessment is questionable. PGA remains an option for treating physicians to contribute their own opinion and experience and to not rely on objective measures only. The use and especially the correct threshold of PGA for remission still has to be discussed.

\section{TREATMENT TARGET IN NEWLY DIAGNOSED SLE PATIENTS: LOW DISEASE ACTIVITY AND REMISSION ARE INDEPENDENTLY ASSOCIATED WITH LOWER ACCRUAL OF EARLY DAMAGE}

Matteo Piga, Alberto Floris, Daniela Perra, Elisabetta Chessa, Mattia Congia, Alessandro Mathieu, Alberto Cauli. Reumatologia, Policlinico Universitario AOU e Università di Cagliari, Cagliari, Italy

\subsection{6/lupus-2020-eurolupus.29}

Background/Purpose To compare the independent effect of achievement and maintenance of lupus low disease activity state (LLDAS) and clinical remission (CR) in preventing early damage accrual in the early stage of systemic lupus erythematosus (SLE) management.

Methods In a monocentric inception cohort of 116 newly diagnosed SLE patients, LLDAS and CR achievement at 6 months (T1) after treatment initiation and their maintenance over the next 12 (T2) months were assessed. Early damage was assessed (T2) using the SLICC/damage index. Uni- and multivariate analysis were performed to evaluate the association of LLDAS and CR with early damage.

Results LLDAS achievement was significantly more frequent than CR both at T1 $(42.2 \%$ vs. $21.6 \%, \mathrm{p}<0.001)$ and T2 (46.6\% vs. $31.9 \%, p=0.022)$, with an increasing trend in the overlap rate observed over follow-up (from $51.0 \%$ at $\mathrm{T} 1$ to $68.5 \%$ at T2). Higher SLEDAI score (OR: 1.33, 95\%CI 1.04 $1.71, \mathrm{p} \mathrm{0.022)}$ at baseline, but not higher prednisone dose, was associated with failure to achieve CR at T1.

The overlap between persistent LLDAS and persistent CR between T1 and T2 was observed in $41.7 \%$ of cases. On multivariate analysis, the achievement of CR (OR 0.1, 95\%CI $0.01-0.59, \mathrm{p}=0.015)$ and LLDAS without CR (OR 0.2; 95\% CI $0.06-0.99, \mathrm{p}=0.049$ ) at $\mathrm{T} 1$, as well as younger age at onset (OR $0.95,95 \%$ CI $0.91-0.98, p=0.004$ ), were negatively associated with early damage. Patients who achieved LLDAS at T1 and steadily persisted in this condition until T2 developed significantly less damage compared to those who failed to maintain it during the T1-T2 interval $(p=0.003)$, those who achieved it later than T1 $(\mathrm{p}<0.001)$ or those who had never been in this condition $(\mathrm{p}<0.001)$.

Conclusions Although $\mathrm{CR}$ is recommended as the primary treatment target in SLE, LLDAS may represent a valid alternative in the early stage of SLE management. LLDAS and CR maintenance should be targeted to prevent damage.

\section{EVOLUTION OF KIDNEY ANTIBODY SECRETING CELLS MOLECULAR SIGNATURE IN LUPUS PATIENTS WITH ACTIVE NEPHRITIS UPON IMMUNOSUPPRESSIVE THERAPY}

\begin{abstract}
1,2 Etienne Crickx, ${ }^{3}$ Farah Tamirou, ${ }^{1,4}$ Tessa Huscenot, ${ }^{5}$ Nathalie Costedoat, ${ }^{6}$ Marion Rabant, ${ }^{7}$ Alexandre Karras, ${ }^{1}$ Tatiana Fadeev, ${ }^{5}$ Véronique Le Guern, ${ }^{8}$ Philippe Remy, ${ }^{9}$ Aurélie Hummel, ${ }^{3}$ Bernard Lauwerys, ${ }^{1}$ Jean-Claude Weill, ${ }^{1}$ Claude-Agnès Reynaud, ${ }^{3}$ Frédéric Houssiau, ${ }^{1,2}$ Matthieu Mahévas. ${ }^{1}$ Institut Necker-Enfants-Malades, INSERMU1151/CNRS-UMS 8253, Université Paris Descartes, Paris; ${ }^{2}$ APHP, Internal Medicine Dept., Henri-Mondor Hospital, Université Paris-Est, Créteil, France; ${ }^{3}$ Service de Rhumatologie, Cliniques Universitaires Saint-Luc, Brussels, Belgium; ${ }^{4}$ APHP, Dept. of Internal Medicine, Lariboisière Hospital, Paris; ${ }^{5}$ APHP, Internal Medicine Dept., Cochin Hospital, Paris; ${ }^{6}$ APHP, Pathology Dept., Necker Hospital, Paris; ${ }^{7}$ APHP, Nephrology Dept., HEGP, Paris; ${ }^{8}$ APHP, Nephrology Dept., Henri-Mondor Hospital, Créteil; ${ }^{9}$ APHP, Nephrology Dept., Necker Hospital, Paris, France
\end{abstract}

10.1136/lupus-2020-eurolupus.30 
Background/Purpose Pathogenic antibody-secreting cells (ASC) are poorly characterized in human lupus nephritis (LN). Our objective was to compare the single cell molecular signature of ASC in kidney and urine from patients with active LN, either untreated or after immunosuppressive therapy failure.

Methods ASC were identified by anti-CD138 staining on fixed renal biopsies from patients with active LN. We sorted singleASC from fresh renal biopsies to perform gene expression profiling. ASC transcriptional program from urine of untreated LN patients was assessed at diagnosis and after a prospective follow up during induction therapy.

Results Interstitial infiltrates of CD138+ ASC were found in untreated $(\mathrm{N}=15)$ and refractory patients $(\mathrm{N}=6)$. Single cell molecular signature of kidney ASC from untreated patients revealed that these cells were mostly plasmablasts and contrasted with ASC signature from patients with mycophenolate mofetil failure that expressed long-lived plasma cells genes and clustered with long-lived bone marrow ASC from healthy donors. A plasmablast signature was observed in urine ASC at diagnosis, similar to their kidney counterpart. The concentration of urine ASC from 22 untreated patients correlated with ISN/RPS classification, with higher concentration in class IV patients $(\mathrm{p}<0.01)$.

Conclusion These results suggest that plasmablasts infiltrate kidney of untreated LN patients, while kidney long-lived ASC may contribute to the failure of immunosuppressive therapy. Acknowledgement This work was funded by FOREUM.

\section{O20 MARGINAL ZONE B CELL DEVELOPMENT FROM EARLY T2 PROGENITORS IS DEFECTIVE IN LUPUS NEPHRITIS}

${ }^{1}$ Thomas J Tull, 'William Guesdon, ${ }^{2}$ Michael G Robson, 'David D'Cruz, ${ }^{3}$ Mats Bemark, ${ }^{1}$ Jo Spencer. 'School of Immunology and Microbial Sciences, King's College London, London; 'Dept. of Nephrology, Guy's and St Thomas' NHS Trust, London, UK; ${ }^{3}$ Dept. of Microbiology and Immunology, University of Gothenburg, Gothenburg, Sweden

10.1136/lupus-2020-eurolupus.31

Background Marginal zone B cells (MZB) are a distinct B cell subset but the site and nature of their differentiation in humans is not understood. In mice, MZB are formed from transitional T2 cells shortly after exit from the bone marrow into the blood. Here we demonstrate a human MZB maturation axis that emerges at the T2 stage that collapses in lupus nephritis (LN).

Methods Mass cytometry was used to phenotype B cells from blood and gut-associated lymphoid tissue (GALT) from healthy donors (HCD). Single cell RNA sequencing was performed using a 10X genomics platform. Flow and mass cytometry were used to investigate MZB differentiation in LN.

Results Deep phenotypic analysis of B cells from HCD revealed prominent $\operatorname{IgM}^{\text {hi }}$ and $\operatorname{IgM}^{\text {lo }}$ TS cell populations emerge at the T2 stage and phenotypically align with $\mathrm{IgM}^{\mathrm{hi}} \mathrm{CD} 45 \mathrm{RB}^{\mathrm{hi}}$ marginal zone precursor cells (MZP). IgM ${ }^{\text {hi }}$ TS cells had high expression of the gut homing receptor $\alpha 4 \beta 7$ integrin and were grossly enriched in GALT. IgM ${ }^{\text {hi }}$ TS $B$ cells produced IL-10 and were enriched in retinoic acid and lipopolysaccharide genes consistent with exposure to the gut microenvironment. MZB depletion was seen in $\mathrm{LN}$ alongside depletion of MZP and TS $\operatorname{IgM}^{\text {hi }}$ cells, suggesting collapse of this maturational axis.

Conclusion We identify a bifurcation of human B cell development that starts at the T2 stage and gives rise to a gut homing $\operatorname{IgM}^{\mathrm{hi}}$ branch that is functionally and phenotypically distinct. The depletion of this branch in patients with LN affirms its existence in health, but also has important disease implications, such as the susceptibility of LN patients to pneumococcal infections and diminished $\mathrm{B}$ regulatory responses seen in the disease.

Acknowledgements This work was funded by the Medical Research Council of Great Britain (MR/R000964/1) and The Lupus Trust.

\section{ANALYSIS OF B-CELL INFILTRATES AND TERTIARY LYMPHOID ORGAN IN LUPUS NEPHRITIS}

Fahd Touzani, Agnieszka Pozdzik. Nephrology Dept., Brugmann Hospital, Brussels, Belgium

\subsection{6/lupus-2020-eurolupus.32}

Background Renal impairment is the leading cause of morbidity and mortality in systemic lupus erythematosus. Tertiary lymphoid organs (TLO) are organized lymphoid structures that develop in response to inflammatory signals from tissues. They may organize into a functional ectopic germinal center. Their pathogenic role in the evolution of kidney function in lupus nephritis remains uncertain.

Method To assess the correlation between tertiary lymphoid organs and B-cell infiltrates and the severity and outcome of kidney function, we conducted a retrospective study of renal biopsy in patients with lupus nephritis. Immunophenotyping of the cell infiltrate was evaluated by immunohistochemistry. We assessed the B-cell infiltrate with a semi-quantitative score. Results 18 adult patient biopsies identified over the 10 -year period met the inclusion criteria.

Immunophenotyping of the inflammatory infiltrate was performed in 17 patients. There was no inflammatory infiltrate in 3 patients. Scattered $\mathrm{T}$ and $\mathrm{B}$ cells were found in 4 patients and $40 \%$ of patients showed organized clusters of $\mathrm{T}$ and $\mathrm{B}$ lymphocytes (grade 3 and 4 infiltration) with TLO in 2 biopsies. 9 of the 18 patients went into complete renal remission. Of the 12 patients with unorganized inflammatory infiltrates (grade $0,1,2), 7$ patients $(58.3 \%)$ achieved recovery. In contrast, 2 out of 5 patients $(40 \%)$ with organized infiltrates (3 and 4$)$ reached complete renal remission $(p=0.44)$. The time to renal remission was longer in patient with unorganized infiltration than those with organized infiltrate (median time over 24 vs. 18.9 months respectively, $\mathrm{p}=0.24$ ) (figure 1). Of

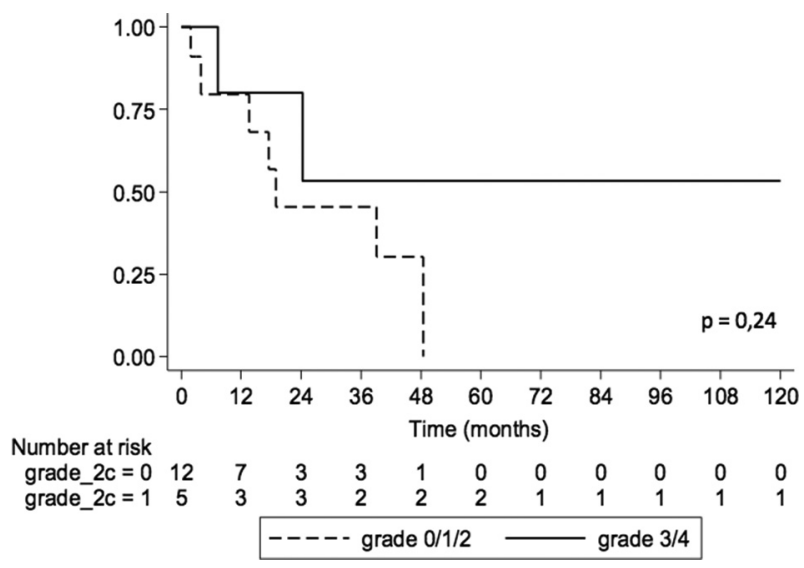

Abstract 021 Figure 1 Kaplan Meier curve of renal remission according to the inflammatory infiltrate 